\title{
Controllability of Linear Passive Network Behaviors
}

\author{
Timothy H. Hughes ${ }^{\mathrm{a}, *}$, Malcolm C. Smith ${ }^{\mathrm{a}}$ \\ ${ }^{a}$ Cambridge University Engineering Department, Trumpington Street, Cambridge, UK, CB2 1PZ
}

\begin{abstract}
Classical RLC realisation procedures (e.g. Bott-Duffin) result in networks with uncontrollable driving-point behaviors. With this motivation, we use the behavioral framework of Jan Willems to provide a rigorous analysis of RLC networks and passive behaviors. We show that the driving-point behavior of a general RLC network is stabilisable, and controllable if the network contains only two types of elements. In contrast, we show that the full behavior of the RLC network need not be stabilisable, but is marginally stabilisable. These results allow us to formalise the phasor approach to RLC networks using the notion of sinusoidal trajectories, and to address an assumption of conventional phasor analysis. Finally, we show that any passive behavior with a hybrid representation is stabilisable. This paper relies substantially on the fundamental work of our late friend and colleague Jan Willems to whom the paper is dedicated.
\end{abstract}

Keywords: Behaviors, Electric circuits, Mechanical networks, Passivity, Controllability, Stabilisability

\section{Introduction}

Traditionally, questions of synthesis for passive networks have been framed in terms of achieving a given transfer function at the driving-point terminals. Brune's seminal contribution [4] was to show that a rational function can be realised as the impedance of a 2-terminal passive network if and only if it is positive-real. Subsequently, the Bott-Duffin procedure [3], and the reactance extraction scheme of Youla and Tissi [18], demonstrated that this result also holds for (two-terminal) RLC networks and multi-port passive networks, respectively. It has long been observed that the Bott-Duffin procedure, and its variants $[12,9,14,5]$, generate RLC networks which contain a greater number of energy storage elements than the McMillan degree of the transfer function to be realised. Nevertheless, it has recently been established that, for the realisation of certain transfer functions, these networks actually contain the least possible number of energy storage elements $[7,6]$. These facts motivate a fundamental treatment of the analysis of passive networks in a manner appropriate to the study of this apparent non-minimality. The behavioral approach and the dissipativity concept of Jan Willems are ideally suited to this task.

One significant contribution of the behavioral approach is a representation-free definition of the concepts 'controllability', 'stabilisability', and 'marginal stabilisability' (see [15, pp. 70-71] and Section 2 of this paper). As emphasised in [11, Section 8.2.3], the transfer function of a system is only sufficient for determining its behavior when that behavior is controllable. Indeed, despite the aforementioned necessary and sufficient conditions on the transfer functions of passive networks, it is still not known what are

* Corresponding author the necessary conditions for a (not necessarily controllable) behavior to be realised as the driving-point behavior of a passive network [16, Section 12]. In this paper, we will derive additional necessary conditions pertaining to the stabilisability of the driving-point behavior and full behavior of an RLC network, and to the controllability of the driving-point behavior of any network which contains only two types of elements (an LC, RC, or RL network).

The structure of the paper, and the key contributions, are as follows: We begin with some notation and preliminaries in Section 2. Throughout Sections 3 to 6, our focus is on RLC networks, which we analyse using a combination of the behavioral framework of Jan Willems with graph theory results from [2]. The approach in these sections is principally algebraic and exploits the correspondence between linear time-invariant differential systems and polynomial modules in the manner of Willems [11, 15]. With these tools, we show that the driving-point behavior of an RLC network is necessarily stabilisable (Theorem 2), and controllable when the network contains only two types of elements (Theorem 3). In contrast, we show that the full behavior of an RLC network need not be stabilisable, but is necessarily marginally stabilisable (Theorem 4). With these results, we formalise the phasor analysis of RLC networks using the idea of sinusoidal trajectories, and address an assumption of conventional phasor analysis (Theorem 5). Then, in Section 7, we investigate the controllability of the driving-point and full behavior of the Bott-Duffin networks. Finally, in Section 8, we adopt a different approach aligned with the dissipativity concept of Willems, particularly the representation-free definition proposed in $[16$, Section 8], in order to study passive behaviors in greater generality. In particular, we show that any passive behavior with a hybrid representation is stabilisable. This result 
is applicable to the terminal behavior of any network comprising an interconnection of the usual passive elements (resistor, capacitor, inductor, transformer, gyrator).

\section{Notation and preliminaries}

In this paper, $\mathbb{R}$ (resp. $\mathbb{C}, \mathbb{Z}$ ) will denote the real (resp. complex, integer) numbers. For $z \in \mathbb{C}, \Re(z)$ denotes its real-part. $\mathbb{C}_{+}$(resp. $\left.\mathbb{C}_{-}, \overline{\mathbb{C}}_{+}, \overline{\mathbb{C}}_{-}\right)$denotes the open right half plane (resp. open left half plane, closed right half plane, closed left half plane). $\mathbb{R}[s]$ and $\mathbb{R}(s)$ will denote the space of polynomials and rational functions with real coefficients, respectively. $\mathbb{R}_{p}(s)$ will denote the proper (i.e. bounded at infinity) rational functions, and $\mathcal{R} \mathcal{H}_{\infty}$ will denote the subspace of $\mathbb{R}_{p}(s)$ containing those functions which are analytic in $\overline{\mathbb{C}}_{+}$. Let $\mathbb{F}$ be one of $\mathbb{R}$, $\mathbb{C}, \mathbb{R}[s], \mathbb{R}(s)$, or $\mathbb{R}_{p}(s)$. Then $\mathbb{F}^{m \times n}$ (resp. $\mathbb{F}^{m}$ ) denotes the matrices with $m$ rows and $n$ columns (resp. vectors with $m$ rows) whose entries are from $\mathbb{F}$, and we write $\mathbb{F}^{\bullet} \bullet \bullet\left(\right.$ resp. $\left.\mathbb{F}^{\bullet}\right)$ when the dimensions are immaterial. $M^{*}$ will denote the Hermitian transpose of $M \in \mathbb{C}^{m \times n}$. For $D \in \mathbb{C}^{m \times m}, D \geq 0$ (resp. $D \leq 0$ ) indicates that $D$ is positive (resp. negative) semidefinite. $I_{m}$ will denote the identity matrix with $m$ rows and $m$ columns, and the dimension will occasionally be omitted when it is clear from the context. Finally, $\operatorname{diag}\left(x_{1} \cdots x_{m}\right)$ will denote the diagonal matrix whose diagonal entries are $x_{1}, \ldots, x_{m}$, and $\operatorname{col}\left(\begin{array}{lll}A_{1} & \cdots & A_{m}\end{array}\right)$ will denote the block column matrix $\operatorname{col}\left(\begin{array}{lll}A_{1} & \cdots & A_{m}\end{array}\right)=\left[\begin{array}{lll}A_{1}^{T} & \cdots & A_{m}^{T}\end{array}\right]^{T}$.

We say that $H \in \mathbb{R}^{n \times n}(s)$ is positive-real (PR) if (i) $H$ is analytic in $\mathbb{C}_{+}$, and (ii) $H(\xi)^{*}+H(\xi) \geq 0$ for all $\xi \in \mathbb{C}_{+}$. Here, (ii) is equivalent to (iia) $H(j \omega)^{*}+H(j \omega) \geq 0$ for all $\omega \in \mathbb{R}$ with $j \omega$ not a pole of any element of $H$, and (iib) any poles of $H$ on $j \mathbb{R} \cup \infty$ are simple and have a positive semidefinite residue matrix [1, Thm 2.7.2].

Throughout this paper, we will consider linear timeinvariant differential behaviors in the sense of [11], which we will frequently describe as the kernel of a differential operator $R\left(\frac{d}{d t}\right)$ for some $R \in \mathbb{R}^{\bullet} \times[\xi]$. We refer to a particular element of the behavior as a trajectory. As in [11], we will consider behaviors to comprise trajectories which are locally integrable, i.e. $\mathcal{B}:=\left\{\mathbf{b} \in \mathcal{L}_{1}^{\text {loc }}\left(\mathbb{R}, \mathbb{R}^{\bullet}\right) \mid\right.$ $\left.R\left(\frac{d}{d t}\right) \mathbf{b}=0\right\}$. Here, differentiation is interpreted in a weak sense, and we identify any two locally integrable functions which are equal except on a set of measure zero (see [11, Section 2.3.2]). We will denote the subset of $\mathcal{B}$ comprising the infinitely differentiable trajectories by $\mathcal{B} \cap \mathcal{C}^{\infty}\left(\mathbb{R}, \mathbb{R}^{\bullet}\right):=\left\{\mathbf{b} \in \mathcal{C}^{\infty}\left(\mathbb{R}, \mathbb{R}^{\bullet}\right) \mid R\left(\frac{d}{d t}\right) \mathbf{b}=0\right\}$, and we note that any trajectory in $\mathcal{B} \cap \mathcal{C}^{\infty}\left(\mathbb{R}, \mathbb{R}^{\bullet}\right)$ is also a solution to $R\left(\frac{d}{d t}\right) \mathbf{b}=0$ in the usual sense [11, Theorem 2.3.11].

If $\tilde{R}=U R$ for some unimodular $U$, then the sets of locally integrable functions in the kernels of $R\left(\frac{d}{d t}\right)$ and $\tilde{R}\left(\frac{d}{d t}\right)$ are identical [11, Theorem 2.5.4]. In particular, this enables the elimination of variables from a behavior $\mathcal{B}:=\left\{\mathbf{b} \in \mathcal{L}_{1}^{\text {loc }}\left(\mathbb{R}, \mathbb{R}^{\bullet}\right) \mid R\left(\frac{d}{d t}\right) \mathbf{b}=0\right\}$. Suppose $\mathbf{b}$ is partitioned as $\mathbf{b}=$ : $\operatorname{col}(\mathbf{d} \mathbf{r})$, and we wish to eliminate $\mathbf{r}$ from $R\left(\frac{d}{d t}\right) \mathbf{b}=: R_{1}\left(\frac{d}{d t}\right) \mathbf{d}+R_{2}\left(\frac{d}{d t}\right) \mathbf{r}=0$. By [11, Theorem 2.5.23], there exists a unimodular $U=\left[\begin{array}{ll}U_{1} & U_{2}\end{array}\right]^{T}$ such that

$$
\left[\begin{array}{c}
U_{1}^{T} \\
U_{2}^{T}
\end{array}\right] R_{1}=\left[\begin{array}{c}
D_{1,1} \\
D_{2,1}
\end{array}\right], \text { and }\left[\begin{array}{c}
U_{1}^{T} \\
U_{2}^{T}
\end{array}\right] R_{2}=\left[\begin{array}{c}
0 \\
D_{2,2}
\end{array}\right]
$$

and where $D_{2,2}(\xi)$ has full row rank (equal to the rank of $R_{2}(\xi)$ ) for almost all $\xi \in \mathbb{C}$. From [10], then the behavior $\mathcal{B}_{d}:=\left\{\mathbf{d} \in \mathcal{L}_{1}^{\text {loc }}\left(\mathbb{R}, \mathbb{R}^{\bullet}\right) \mid \exists \mathbf{r} \in \mathcal{L}_{1}^{\text {loc }}\left(\mathbb{R}, \mathbb{R}^{\bullet}\right)\right.$ which satisfies $\left.R_{1}\left(\frac{d}{d t}\right) \mathbf{d}+R_{2}\left(\frac{d}{d t}\right) \mathbf{r}=0\right\}$ is equal to the set of solutions to $D_{1,1}\left(\frac{d}{d t}\right) \mathbf{d}=0$ which satisfy certain smoothness conditions. In some cases $\mathcal{B}_{d}=\left\{\mathbf{d} \in \mathcal{L}_{1}^{\text {loc }}\left(\mathbb{R}, \mathbb{R}^{\bullet}\right) \mid D_{1,1}\left(\frac{d}{d t}\right) \mathbf{d}=\right.$ $0\}$, in which case we call $r$ properly eliminable (see [10, Theorems 2.5 and 2.8], which contain criteria for proper eliminability).

A behavior $\mathcal{B}$ is called controllable if for any two trajectories $\mathbf{b}_{1}, \mathbf{b}_{2} \in \mathcal{B}$, there exists a $t_{1} \geq 0$ and $\mathbf{a} \mathbf{b} \in \mathcal{B}$ which satisfies $\mathbf{b}(t)=\mathbf{b}_{1}(t)$ for all $t \leq 0$ and $\mathbf{b}(t)=\mathbf{b}_{2}(t)$ for all $t \geq t_{1}$ [11, Definition 5.2.2]. It is called stabilisable if for every $\mathbf{b}_{1} \in \mathcal{B}$, there exists $\mathbf{a} \mathbf{b} \in \mathcal{B}$ which satisfies $\mathbf{b}(t)=\mathbf{b}_{1}(t)$ for all $t \leq 0$ and $\lim _{t \rightarrow \infty} \mathbf{b}(t)=0$; and marginally stabilisable if for every $\mathbf{b}_{1} \in \mathcal{B}$, there exists a $t_{1} \geq 0$ and $\mathbf{a} \mathbf{b} \in \mathcal{B}$ which satisfies $\mathbf{b}(t)=\mathbf{b}_{1}(t)$ for all $t \leq 0$ and $\mathbf{b}(t)$ is bounded in $t \geq t_{1}$. From [11, Thms 5.2.10 and 5.2.30], whenever $R \in \mathbb{R}^{\bullet} \times[s]$, then the behavior $\mathcal{B}:=\left\{\mathbf{b} \in \mathcal{L}_{1}^{\text {loc }}\left(\mathbb{R}, \mathbb{R}^{\bullet}\right) \mid R\left(\frac{d}{d t}\right) \mathbf{b}=0\right\}$ is controllable (resp. stabilisable) if and only if the rank of $R(\xi)$ is the same for all $\xi \in \mathbb{C}$ (resp. $\xi \in \overline{\mathbb{C}}_{+}$).

A numbered list containing further relevant material is provided in Section 10. The items in this list will be referred to in the text when needed, with a superscript used to indicate the number of the relevant list item.

\section{RLC networks and behaviors}

In this section, we present explicit and parsimonious descriptions of the full behavior and the driving-point behavior of a given RLC network.

We define resistors, inductors and capacitors as the idealised elements shown in Fig. 1. Each such element is associated with a current $i \in \mathcal{L}_{1}^{\text {loc }}(\mathbb{R}, \mathbb{R})$ through the element and a voltage $v:=v^{+}-v^{-} \in \mathcal{L}_{1}^{\text {loc }}(\mathbb{R}, \mathbb{R})$ across the element which are constrained to satisfy the corresponding differential equation given in that figure. We remark that, by identifying force with current and velocity with voltage, there is a direct analogy between RLC networks and mechanical networks comprising dampers, springs, and inerters (see Fig. 1). Consequently, the conclusions of this paper are equally applicable to the analysis of such mechanical networks.

In Sections 3 to 6 , we restrict attention to RLC 'oneport' networks. Any such network $N$ has the structure of a connected, oriented graph ${ }^{1}$ (hereafter referred to as a graph) with vertices $x_{1}, \ldots x_{n}$ and edges $y_{1}, \ldots, y_{m}$, and with two designated external vertices corresponding to the two terminals which constitute the port of $N$. Each edge 


\begin{tabular}{|c|c|}
\hline Mechar & Electrical \\
\hline 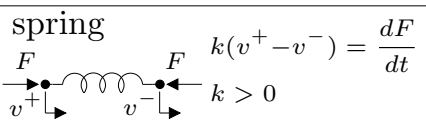 & 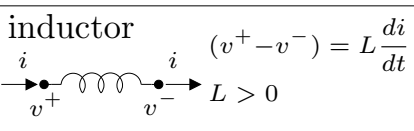 \\
\hline 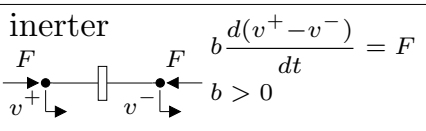 & 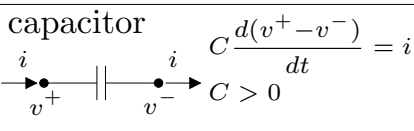 \\
\hline 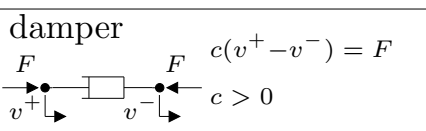 & $\stackrel{v^{+}}{\text {resistor }} \underset{v^{-}}{\stackrel{i}{\rightarrow}} \rightarrow \begin{array}{l}\left(v^{+}-v^{-}\right)=R i \\
R>0\end{array}$ \\
\hline
\end{tabular}

Figure 1: Passive electrical and mechanical elements.

$y_{k}$ represents an element $N_{k}(k=1, \ldots, m)$ which is either a resistor, an inductor, or a capacitor. If all edges correspond to either inductors or capacitors (resp. resistors or capacitors, resistors or inductors) then we call $N$ an LC (resp. RC, RL) network. The edges are oriented so that the current $i_{k}$ through $N_{k}$ is from tail to head, and $\int_{t_{0}}^{t_{1}} i_{k}(t) v_{k}(t) d t$ is the energy supplied to $N_{k}$ between $t_{0}$ and $t_{1}$. Here, $i_{k}$ and $v_{k}$ satisfy the differential equation $p_{k}\left(\frac{d}{d t}\right) i_{k}=q_{k}\left(\frac{d}{d t}\right) v_{k}$ for some coprime $p_{k}, q_{k} \in \mathbb{R}[s]$ corresponding to the element $N_{k}$ (see Fig. 1). An extra edge may be added between the external vertices of $G$, with an associated current $-i$ and voltage $v$, to form a graph $G^{\prime}$ with $n$ vertices and $m+1$ edges. This corresponds to the connection of a source to the port of $N$ as in Fig. 2, and the sign conventions are such that $i$ represents the current flowing through $N$, and $\int_{t_{0}}^{t_{1}} i(t) v(t) d t$ represents the energy supplied to $N$ between $t_{0}$ and $t_{1}$.

With the notation:

$$
\begin{aligned}
& \mathbf{b}:=\operatorname{col}(\mathbf{d} \mathbf{r}), \\
& \text { with } \mathbf{d}:=\operatorname{col}(i v) \text {, } \\
& \text { and } \mathbf{r}:=\operatorname{col}\left(\begin{array}{llllll}
i_{1} & \cdots & i_{m} & v_{1} & \cdots & v_{m}
\end{array}\right) \text {, }
\end{aligned}
$$

we call b a full trajectory (or, simply, trajectory) of the network, and $\mathbf{d}$ a driving-point trajectory of the network, if $\mathbf{b} \in \mathcal{L}_{1}^{\text {loc }}\left(\mathbb{R}, \mathbb{R}^{2 m+2}\right)$, and if the currents and voltages in $\mathbf{b}$ satisfy Kirchhoff's current and voltage laws and the differential equations corresponding to the elements in the network. We then call the set of all trajectories the full behavior (or, simply, behavior) of the network, and the set of all driving-point trajectories will be called the drivingpoint behavior.

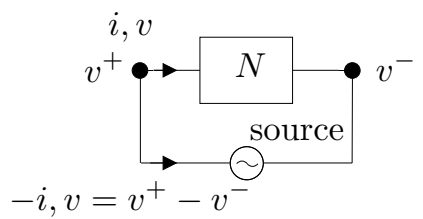

Figure 2: RLC network with source.
The graph $G^{\prime}$ allows certain matrices (and associated vector spaces) to be constructed with a close relationship to Kirchhoff's laws. Let $\tilde{G}$ be a tree in $G^{\prime},{ }^{2}$ and let the edges in $G^{\prime}$ be ordered so that the edges in $\tilde{G}$ are in the last $n-1$ places. Removing one of the edges from $\tilde{G}$ separates the vertices of $G^{\prime}$ into two distinct sets. A cut-set can then be constructed from the single edge that is removed and some of the edges from the complementary chord-set. A corresponding cut-set vector can be defined in which an element of the vector is zero if the corresponding edge does not belong to the cut-set, and \pm 1 otherwise, with the sign chosen according to the orientation of the edge in relation to the two sets of vertices (these are the fundamental cut vectors described in [2, p. 37]). Let $\hat{B}$ be the matrix whose rows are the cut-set vectors comprising a single edge from $\tilde{G}$, taking the edges in turn, so that $\hat{B}$ takes the form

$$
\hat{B}=\left[\begin{array}{ll}
B & I_{n-1}
\end{array}\right] \text {. }
$$

We call $\hat{B}$ a fundamental cut-set matrix, and we call the $\mathbb{R}$-vector space spanned by the rows of $\hat{B}$ the cut-set space.

Now, consider again the tree $\tilde{G}$ and the edges of $G^{\prime}$ ordered as above. Since any tree contains a unique path between any two vertices then, for any edge from the complementary chord-set, there is a unique circuit in $G^{\prime}$ containing just this one edge from the chord-set (together with edges from the tree). A corresponding circuit vector can be defined in which an element of the vector is zero if the corresponding edge does not belong to the circuit, and \pm 1 otherwise, with the sign chosen according to the orientation of the edge in relation to the orientation of the circuit (these are the fundamental cycle vectors described in $[2, \mathrm{p}$. $37])$. Let $\hat{C}$ be the matrix whose rows are circuit vectors comprising a single edge from the complementary chordset, taking the edges in turn, so that $\hat{C}$ takes the form

$$
\hat{C}=\left[\begin{array}{ll}
I_{m+2-n} & C^{T}
\end{array}\right]
$$

We call $\hat{C}$ a fundamental circuit matrix, and we call the $\mathbb{R}$-vector space spanned by the rows of $\hat{C}$ the circuit space. It can be shown that cut-set and circuit vectors are always orthogonal $\left[2\right.$, p. 36], which implies that $\hat{B} \hat{C}^{T}=0$, and hence $B=-C$. Now, let $\mathbf{i} \in \mathcal{L}_{1}^{\text {loc }}\left(\mathbb{R}, \mathbb{R}^{m+1}\right)$ and $\mathbf{v} \in \mathcal{L}_{1}^{\text {loc }}\left(\mathbb{R}, \mathbb{R}^{m+1}\right)$ denote the vectors of edge currents and voltages, in the given edge ordering, for some network trajectory. Then Kirchhoff's current law implies that $\hat{B} \mathbf{i}=0$. Similarly, Kirchhoff's voltage law implies that $\hat{C} \mathbf{v}=0 .^{3}$

Since the graph $G$ is connected and contains the same vertices as $G^{\prime}$, then either (i) $G$ contains two distinct vertices incident with a single edge corresponding to the source, or (ii) $G$ contains a tree $\tilde{G}$ which is also a tree in $G^{\prime}$ (note that $\tilde{G}$ is empty when $G$ contains only one vertex, i.e. $n=1)$. In case (i), there is a single cut-set comprising the one edge in $G$, and $G$ contains no circuits, whence the driving-point behavior has $i=0$ and $v$ free. Consequently, we will not consider this case any further in this paper. In case (ii), we let the edges of $G^{\prime}$ be ordered with the edge 
containing the source in first place, followed by the remaining edges in the complementary chord-set to $\tilde{G}$, and finally the $n-1$ edges from $\tilde{G}$. Let $\mathbf{i}_{1}, \mathbf{v}_{1}$ denote the edge currents and voltages for the elements in the chord-set and let $\mathbf{i}_{2}, \mathbf{v}_{2}$ be those for the elements of the tree. It follows that Kirchhoff's voltage law takes the form

$$
\left[\begin{array}{ccc}
1 & 0 & C_{1}^{T} \\
0 & I_{m+1-n} & C_{2}^{T}
\end{array}\right]\left[\begin{array}{c}
v \\
\mathbf{v}_{1} \\
\mathbf{v}_{2}
\end{array}\right]=0
$$

and Kirchhoff's current law takes the form

$$
\left[\begin{array}{lll}
-C_{1} & -C_{2} & I_{n-1}
\end{array}\right]\left[\begin{array}{c}
-i \\
\mathbf{i}_{1} \\
\mathbf{i}_{2}
\end{array}\right]=0
$$

Evidently, $C_{1}=0$ if and only if the two driving-point vertices are identical, and if $C_{1}=0$ then the driving-point behavior has $v=0$ and $i$ free. Consequently, in the remainder of this paper, we consider the two driving-point vertices to be distinct, and hence $C_{1} \neq 0$ (and, in particular, $n>1)$. It remains possible that $m+1-n=0$, in which case the terms involving $\mathbf{i}_{1}, \mathbf{v}_{1}$, and $C_{2}$ are removed from the preceding equations.

We first consider the case $m+1-n>0$, and we define the diagonal matrices $P_{1}:=\operatorname{diag}\left(p_{1} \cdots p_{m+1-n}\right), Q_{1}:=$ $\operatorname{diag}\left(q_{1} \cdots q_{m+1-n}\right), P_{2}:=\operatorname{diag}\left(p_{m+2-n} \cdots p_{m}\right)$, and $Q_{2}:=\operatorname{diag}\left(q_{m+2-n} \cdots q_{m}\right)$. The full behavior of the network is then the set of $\mathbf{b} \in \mathcal{L}_{1}^{\text {loc }}\left(\mathbb{R}, \mathbb{R}^{2 m+2}\right)$ as in (2) which satisfy (5) and (6) and the equations $P_{1}\left(\frac{d}{d t}\right) \mathbf{i}_{1}=$ $Q_{1}\left(\frac{d}{d t}\right) \mathbf{v}_{1}$ and $P_{2}\left(\frac{d}{d t}\right) \mathbf{i}_{2}=Q_{2}\left(\frac{d}{d t}\right) \mathbf{v}_{2}$. Thus, up to a reordering of the variables, we obtain a kernel representation for the network's behavior:

$$
\left[\begin{array}{cccccc}
0 & 1 & 0 & C_{1}^{T} & 0 & 0 \\
0 & 0 & P_{1} & 0 & -Q_{1} & 0 \\
0 & 0 & 0 & Q_{2} & 0 & -P_{2} \\
0 & 0 & 0 & C_{2}^{T} & I & 0 \\
C_{1} & 0 & -C_{2} & 0 & 0 & I
\end{array}\right] \quad\left(\frac{d}{d t}\right)\left[\begin{array}{c}
i \\
v \\
\mathbf{i}_{1} \\
\mathbf{v}_{2} \\
\mathbf{v}_{1} \\
\mathbf{i}_{2}
\end{array}\right]=0
$$

The driving-point behavior of the network $\mathcal{B}_{d}$ is then the set of $\operatorname{col}(i v) \in \mathcal{L}_{1}^{\text {loc }}\left(\mathbb{R}, \mathbb{R}^{2}\right)$ for which there exists a $\operatorname{col}\left(\begin{array}{llll}\mathbf{i}_{1} & \mathbf{v}_{2} & \mathbf{v}_{1} & \mathbf{i}_{2}\end{array}\right) \in \mathcal{L}_{1}^{\text {loc }}\left(\mathbb{R}, \mathbb{R}^{2 m}\right)$ satisfying $(7)$. That $\mathcal{B}_{d}$ is the set of locally integrable functions in the kernel of a differential operator follows from the following theorem, whose proof is postponed until Section 6 as it is a simple extension to the proof of Theorem 5 .

Theorem 1. The driving-point behavior of any RLC network takes the form

$$
\mathcal{B}_{d}:=\left\{\left[\begin{array}{l}
i \\
v
\end{array}\right] \in \mathcal{L}_{1}^{l o c}\left(\mathbb{R}, \mathbb{R}^{2}\right) \mid D_{1,1}\left(\frac{d}{d t}\right)\left[\begin{array}{l}
i \\
v
\end{array}\right]=0\right\},
$$

for some $D_{1,1} \in \mathbb{R}^{\bullet \times \bullet}[s]$.

We now follow the procedure outlined in Section 2 to obtain an explicit description for the differential operator
$D_{1,1}\left(\frac{d}{d t}\right)$ in Theorem 1 from the kernel representation (7). We first define the diagonal polynomial matrices:

$$
\begin{aligned}
F & :=\operatorname{diag}\left(\begin{array}{llllll}
q_{1} & \cdots & q_{m+1-n} & p_{m+2-n} & \cdots & p_{m}
\end{array}\right), \\
\text { and } \quad G & :=\operatorname{diag}\left(\begin{array}{llllll}
p_{1} & \cdots & p_{m+1-n} & q_{m+2-n} & \cdots & q_{m}
\end{array}\right),
\end{aligned}
$$

so $F=\operatorname{diag}\left(Q_{1} P_{2}\right)$ and $G=\operatorname{diag}\left(P_{1} Q_{2}\right)$. Next, we define the real-valued matrices:

$$
H:=\left[\begin{array}{cc}
0 & C_{2}^{T} \\
-C_{2} & 0
\end{array}\right], \text { and } x:=\left[\begin{array}{c}
0 \\
C_{1}
\end{array}\right]
$$

The polynomial matrix in (7) then takes the form

$$
W=\left[\begin{array}{cccc}
0 & 1 & x^{T} & 0 \\
0 & 0 & G & -F \\
x & 0 & H & I
\end{array}\right]
$$

Then, with $K:=\operatorname{col}(0-F) \in \mathbb{R}^{(m+1) \times m}$ and $L:=$ $\left[\begin{array}{lll}x & 0 & H\end{array}\right] \in \mathbb{R}^{m \times(m+2)}$, we find that

$$
W=\left[\begin{array}{cc}
I & K \\
0 & I
\end{array}\right]\left[\begin{array}{cc}
R & 0 \\
L & I
\end{array}\right]
$$

where $R$ is defined as

$$
R:=\left[\begin{array}{ccc}
0 & 1 & x^{T} \\
F x & 0 & F A
\end{array}\right], \text { with } A:=F^{-1} G+H .
$$

Since the first matrix on the right hand side of (10) is unimodular, we find that the behavior of the network is determined by the behavior:

$\mathcal{B}_{r}:=\left\{\left[\begin{array}{c}i \\ v \\ \mathbf{i}_{1} \\ \mathbf{v}_{2}\end{array}\right] \in \mathcal{L}_{1}^{\text {loc }}\left(\mathbb{R}, \mathbb{R}^{m+2}\right) \mid R\left(\frac{d}{d t}\right)\left[\begin{array}{c}i \\ v \\ \mathbf{i}_{1} \\ \mathbf{v}_{2}\end{array}\right]=0\right\}$,

and, given $\operatorname{col}\left(\begin{array}{llll}i & v & \mathbf{i}_{1}^{T} & \mathbf{v}_{2}^{T}\end{array}\right) \in \mathcal{B}_{r}, \mathbf{v}_{1}$ and $\mathbf{i}_{2}$ are uniquely determined by the linear equations corresponding to the final two block rows in (7).

To describe $D_{1,1}\left(\frac{d}{d t}\right)$ in Theorem 1 explicitly, it remains to eliminate $\mathbf{i}_{1}$ and $\mathbf{v}_{2}$ from $\mathcal{B}_{r}$. Following Section 2 , we let $R_{1}$ and $R_{2}$ in (1) be:

$$
R_{1}:=\left[\begin{array}{cc}
0 & 1 \\
F x & 0
\end{array}\right], \text { and } R_{2}:=\left[\begin{array}{c}
x^{T} \\
F A
\end{array}\right]
$$

and we seek a $U_{1} \in \mathbb{R}^{(m+1) \times(m+1-r)}[s]$ such that $U_{1}^{T} R_{2}=$ 0 , and $U_{1}(\xi)^{T}$ has full row rank (equal to $m+1-r$ ) for all $\xi \in \mathbb{C}$, where $r$ is the rank of $R_{2}(\xi)$ for almost all $\xi \in \mathbb{C}$. Then, in Theorem 1 , we have $D_{1,1}=U_{1}^{T} R_{1}$.

We note that both $F$ and $G$ are invertible, that $F$ and $G$ are coprime with $F^{-1} G$ diagonal and $\mathrm{PR}$, and that $H^{*}=-H$. Thus, $A$ is analytic in $\mathbb{C}_{+}$, and $A(\xi)+A(\xi)^{*}=$ $F^{-1} G(\xi)+F^{-1} G(\xi)^{*}>0$ for all $\xi \in \mathbb{C}_{+}$, and we conclude that $A$ is $\mathrm{PR}$ and invertible and $A^{-1}$ is $\mathrm{PR}[8$, Theorem 5.8]. Now, let

$$
x^{T} A^{-1} F^{-1}=r^{-1} y^{T},
$$


where $r \in \mathbb{R}[s]$ and $y \in \mathbb{R}^{m}[s]$ are coprime (e.g., let $r$ be the monic least common multiple of all the denominator polynomials of $\left.x^{T} A^{-1} F^{-1}\right)$. In particular, $\left[\begin{array}{ll}r & -y^{T}\end{array}\right] R_{2}=$ 0 , and $\left[\begin{array}{ll}r & -y^{T}\end{array}\right](\xi)$ has rank one for all $\xi \in \mathbb{C}$. Then, since $R_{2} \in \mathbb{R}^{(2 m+1) \times 2 m}[s]$ and $R_{2}(\xi)$ has rank $2 m$ for almost all $\xi \in \mathbb{C}$, we may let $U_{1}^{T}=\left[\begin{array}{ll}r & -y^{T}\end{array}\right]$. Thus, the drivingpoint behavior of $N$ takes the form of (8) where $D_{1,1}=$ $U_{1}^{T} R_{1}=\left[\begin{array}{ll}-y^{T} F x & r\end{array}\right] .^{4}$

Finally, in the case $m+1-n=0, \operatorname{col}\left(i v \mathbf{v}_{2}\right)$ is in the kernel of $R\left(\frac{d}{d t}\right)$ where $R$ is as in (11) with $F:=P_{2}$, $G:=Q_{2}, H:=0$, and $x:=C_{1}$, and $\mathbf{i}_{2}$ is then determined from $\mathbf{i}_{2}=-C_{1} i$. The analysis then proceedes identically. Accordingly, we will only present proofs for the case $m+$ $1-n>0$ in the remainder of this paper.

\section{Controllability of RLC network behaviors}

In this section, we investigate the controllability and stabilisability of the full behavior and the driving-point behavior of an RLC network, and we obtain the following principal results:

Theorem 2. The driving-point behavior $\mathcal{B}_{d}$ of any $R L C$ network is stabilisable. In particular, $\mathcal{B}_{d}$ takes the form of (8) with $D_{1,1}\left(\frac{d}{d t}\right)=f\left(\frac{d}{d t}\right)\left[-p\left(\frac{d}{d t}\right) \quad q\left(\frac{d}{d t}\right)\right]$ for some $f, p, q \in \mathbb{R}[s]$, where $p$ and $q$ are coprime and all of the roots of $f$ are in $\mathbb{C}_{-}$.

Theorem 3. The driving-point behavior $\mathcal{B}_{d}$ of any $L C$, $R C$, or $R L$ network is controllable. In particular, $\mathcal{B}_{d}$ takes the form of (8) with $D_{1,1}\left(\frac{d}{d t}\right)=\left[-p\left(\frac{d}{d t}\right) \quad q\left(\frac{d}{d t}\right)\right]$ for some coprime $p, q \in \mathbb{R}[s]$.

Theorem 4. The full behavior $\mathcal{B}$ of any $R L C$ network is marginally stabilisable. More specifically, with $\mathbf{b}$ as in (2), then $\mathcal{B}=\left\{\mathbf{b} \in \mathcal{L}_{1}^{\text {loc }}\left(\mathbb{R}, \mathbb{R}^{2 m+2}\right) \mid W\left(\frac{d}{d t}\right) \mathbf{b}=0\right\}$ for some $W \in \mathbb{R}^{(2 m+1) \times(2 m+2)}[s]$, where all of the roots of the invariant polynomials of $W$ are in $\overline{\mathbb{C}}_{-}$and those roots on $j \mathbb{R}$ have multiplicity one.

To illustrate these theorems, we consider the three networks in Fig. 3. From Section 3, it may be verified that the driving-point behaviors of these networks have the kernel representations indicated in Fig. 3. It follows that the impedance of each network is equal to 1 . However, the driving-point behavior of the network on the left is controllable, that of the central network is stabilisable but not controllable, and that of the network on the right is not stabilisable. The network on the right is not an RLC network as the rightmost resistor has a negative resistance.

The remainder of this section contains the proofs of Theorems 2 to 4 . We provide an algebraic argument using the explicit descriptions of the behavior and driving-point behavior of an RLC network which were provided in Section 3. In Section 8, we show that Theorem 2 generalises to multi-terminal passive networks by providing an alternative proof based on energy arguments (see Theorem 6).

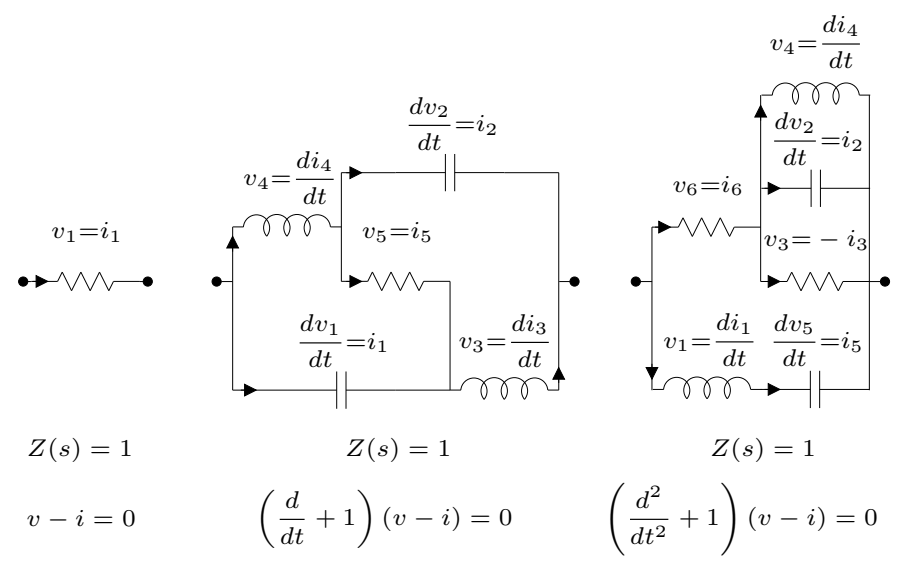

Figure 3: Three networks with the same impedance but different driving-point behaviors.

Indeed, for any given RLC network $N$, Tellegen's theorem (see note 3 ) implies $\int_{t_{0}}^{t_{1}} i(t) v(t) d t=\int_{t_{0}}^{t_{1}} \sum_{k=1}^{m} i_{k}(t) v_{k}(t) d t=$ $\sum_{k=1}^{m} \int_{t_{0}}^{t_{1}} R_{k} i_{k}(t)^{2} d t+(1 / 2)\left[L_{k} i_{k}(t)^{2}+C_{k} v_{k}(t)^{2}\right]_{t_{0}}^{t_{1}}$, hence $\int_{t_{0}}^{t_{1}} i(t) v(t) d t \geq-(1 / 2) \sum_{k=1}^{m} L_{k} i_{k}\left(t_{0}\right)^{2}+C_{k} v_{k}\left(t_{0}\right)^{2}$ for all $t_{1} \geq t_{0}$. Here, $R_{k}$ denotes the resistance, $L_{k}$ the inductance, and $C_{k}$ the capacitance of the element $N_{k}$ (so at most one of $R_{k}, L_{k}$, or $C_{k}$ is non-zero according to whether $N_{k}$ is a resistor, inductor, or capacitor). In other words, the net energy which can be extracted from $N$ cannot exceed the initial energy stored in the inductors and capacitors within $N$. In the proof of Theorem 6 , we show that there is no limit to the net energy which can be extracted from $N$ if the driving-point behavior of $N$ is not stabilisable, and we conclude that the driving-point behavior of any RLC network must be stabilisable.

We also note that the full behavior of an RLC network $N$ need not be stabilisable. Indeed, consider the differential operator $W\left(\frac{d}{d t}\right)$ in $(7)$ corresponding to the central network in Fig. 3. It may be verified that the rank of $W(\xi)$ is equal to eleven for almost all $\xi \in \mathbb{C}$, yet from the discussion which will follow Theorem 5 it will be apparent that the rank of $W(j)$ is ten. Hence, the full behavior of this network is not stabilisable. This loss in stabilisability results from sinusoidal trajectories which can occur even in the absence of any driving-point current or voltage. From Tellegen's theorem (see, again, note 3 ), it follows that these trajectories correspond to energy being exchanged between the inductors and capacitors in $N$, with no current through or voltage across the resistors in $N$.

Proof (Theorem 2). We will use the explicit description of the driving-point behavior $\mathcal{B}_{d}$ from Section 3. Suppose that $\mathcal{B}_{d}$ is not stabilisable. Then, from Section 2, there exists a $\xi \in \overline{\mathbb{C}}_{+}$such that $r(\xi)=0$ and $y^{T} F x(\xi)=0$. Since $r$ and $y$ are coprime then it follows from (14) that, for $\xi \in \mathbb{C}, r(\xi)=0$ if and only if $x^{T} A^{-1} F^{-1}$ has a pole at $\xi$, and whenever $r(\xi)=0$ then the pole of $x^{T} A^{-1} F^{-1}$ and root of $r$ at $\xi$ have equal multiplicities. We will show the 
following two conditions.

(i) $A^{-1}, F^{-1}$, and $A^{-1} F^{-1}$ have no poles in $\mathbb{C}_{+}$, and poles on $j \mathbb{R}$ are simple.

(ii) For $\omega \in \mathbb{R}$, if $x^{T} A^{-1} x$ does not have a pole at $j \omega$, then $x^{T} A^{-1} F^{-1}$ does not have a pole at $j \omega$.

From (i), it follows that $x^{T} A^{-1} F^{-1}$ has no poles in $\mathbb{C}_{+}$, thus $r$ has no roots there. Moreover, for $\omega \in \mathbb{R}$, if $r(j \omega)=$ 0 then $\hat{r}(s):=r(s) /(s-j \omega)$ satisfies $\hat{r}(j \omega) \neq 0$. Now, suppose that $r(j \omega)=0$ and $y^{T} F x(j \omega)=0$. Since, from (14), $y^{T} F x=r x^{T} A^{-1} x$, then it follows that $y^{T} F x(j \omega)=$ $\hat{r}(j \omega) \lim _{s \rightarrow j \omega}\left((s-j \omega) x^{T} A^{-1} x(s)\right)=0$ implies $x^{T} A^{-1} x$ does not have a pole at $j \omega$, hence $x^{T} A^{-1} F^{-1}$ does not have a pole at $j \omega$ by condition (ii). From (14) and condition (i), $y^{T}(j \omega)=\hat{r}(j \omega) \lim _{s \rightarrow j \omega}\left((s-j \omega) x^{T} A^{-1} F^{-1}(s)\right)=0$. We conclude that $r(\xi) \neq 0$ for $\xi \in \mathbb{C}_{+}$and that $r(j \omega)=0$ and $y^{T} F x(j \omega)=0$ imply $y(j \omega)=0$ for any given $\omega \in \mathbb{R}$, which is not possible since $r$ and $y$ are coprime. It follows that any common roots of $y^{T} F x$ and $r$ are in $\mathbb{C}_{-}$, which implies that $\mathcal{B}_{d}$ is stabilisable.

To see condition (i), we recall from Section 3 that $A=$ $F^{-1} G+H$ with $F, G$, and $H$ as defined in that section. Moreover, $A^{-1}$ is $\mathrm{PR}$, whence $A^{-1}$ has no poles in $\mathbb{C}_{+}$, and poles on $j \mathbb{R}$ are simple. Furthermore, $F$ and $G$ are coprime polynomial matrices with $F^{-1}(s)=U_{-1} s^{-1}+$ $U_{0}$ and $F^{-1} G(s)=V_{-1} s^{-1}+V_{0}+V_{1} s$ for some real and diagonal matrices $U_{-1}, U_{0}, V_{-1}, V_{0}, V_{1}$ with $V_{-1}, V_{0}, V_{1} \geq$ 0 . Hence, all the poles of $F^{-1}$ are simple and at the origin. Moreover, $F^{-1} G$ can only have a simple pole at the origin, and there exists a (nonsingular) matrix $T$ such that

$$
\lim _{s \rightarrow 0}\left(s F^{-1}(s)\right)=\lim _{s \rightarrow 0}\left(s F^{-1} G(s)\right) T .
$$

To prove (i), it remains to show that if $A^{-1} F^{-1}$ has a pole at the origin then it is simple. This amounts to showing that $\lim _{s \rightarrow 0}\left(s A^{-1}(s)\right) \lim _{s \rightarrow 0}\left(s F^{-1}(s)\right)=0$. To show this, we note that $I=A^{-1} A=A^{-1} F^{-1} G+A^{-1} H$, whence poles of $A^{-1} F^{-1} G$ at the origin are simple. We conclude that $\lim _{s \rightarrow 0}\left(s A^{-1}(s)\right) \lim _{s \rightarrow 0}\left(s F^{-1} G(s)\right)=0$, which by (15) implies that $\lim _{s \rightarrow 0}\left(s A^{-1}(s)\right) \lim _{s \rightarrow 0}\left(s F^{-1}(s)\right)=0$.

For (ii), we first note that if $x^{T} A^{-1} x$ does not have a pole at $j \omega$ then neither does $x^{T} A^{-1}$. This follows since $A^{-1}$ is PR, so if $A^{-1}$ has a pole at $j \omega$ then it is simple and $W_{-1}:=\lim _{s \rightarrow j \omega}\left((s-j \omega) A^{-1}(s)\right) \geq 0$. Thus, if $x^{T} A^{-1} x$ does not have a pole at $j \omega$, then $x^{T} W_{-1} x=0$, which implies $x^{T} W_{-1}=0$ since $W_{-1} \geq 0$, and hence $x^{T} A^{-1}$ does not have a pole at $j \omega$. Since, in addition, all the poles of $F^{-1}$ are at zero and are simple, then it remains to show (ii) at the origin. As $x^{T} A^{-1}$ does not have a pole at the origin when $x^{T} A^{-1} x$ does not have a pole at the origin, then this amounts to showing that $x^{T} A^{-1}(0) \lim _{s \rightarrow 0}\left(s F^{-1}(s)\right)=0$ when $x^{T} A^{-1}$ does not have a pole at the origin. We note that $x^{T}=x^{T} A^{-1} A=x^{T} A^{-1} F^{-1} G+x^{T} A^{-1} H$, whence if $x^{T} A^{-1}$ does not have a pole at the origin then neither does $x^{T} A^{-1} F^{-1} G$. We therefore conclude that $x^{T} A^{-1}(0) \lim _{s \rightarrow 0}\left(s F^{-1} G(s)\right)=0$, and thus from (15) we obtain $x^{T} A^{-1}(0) \lim _{s \rightarrow 0}\left(s F^{-1}(s)\right)=0$.
Proof (Theorem 3). In this case, we will show the following two conditions.

(i) All of the poles of $x^{T} A^{-1} x$ and $x^{T} A^{-1} F^{-1}$ in $\mathbb{C}_{-}$ are simple.

(ii) For $\xi \in \mathbb{C}_{-}$, if $x^{T} A^{-1} x$ does not have a pole at $\xi$, then $x^{T} A^{-1} F^{-1}$ does not have a pole at $\xi$.

Then, for $\xi \in \mathbb{C}_{-}$, a similar argument to the first paragraph in Theorem 2 shows that we cannot have $r(\xi)=0$ and $y^{T} F x(\xi)=0$. Since, from Theorem 2, this is also true for $\xi \in \overline{\mathbb{C}}_{+}$, then $r$ and $y^{T} F x$ are coprime. This implies that $\mathcal{B}_{d}$ is controllable (see Section 2).

We note that the Schur complement of $Q_{1}^{-1} P_{1}$ in $A$ is

$$
M:=P_{2}^{-1} Q_{2}+C_{2} P_{1}^{-1} Q_{1} C_{2}^{T},
$$

which is invertible since $A$ and $Q_{1}^{-1} P_{1}$ are. We thus obtain

$$
\begin{aligned}
x^{T} A^{-1} & =C_{1}^{T} M^{-1}\left[C_{2} P_{1}^{-1} Q_{1} \quad I\right], \\
\text { and } x^{T} A^{-1} x & =C_{1}^{T} M^{-1} C_{1} .
\end{aligned}
$$

We also recall that all of the poles of $F^{-1}$ and $P_{1}^{-1}$ are at the origin. For an LC network, we will show that all of the poles of $C_{1}^{T} M^{-1}$ are on $j \mathbb{R} \cup \infty$, whence (i) and (ii) are trivially satisfied. Then, for an RC (resp. RL) network, we will show that all of the poles of $M^{-1}$ in $\mathbb{C}_{-}$ are simple and have positive (resp. negative) semidefinite residue matrices. This implies that, for $\xi \in \mathbb{C}_{-}, C_{1}^{T} M^{-1}$ does not have a pole at $\xi$ if $C_{1}^{T} M^{-1} C_{1}$ does not have a pole at $\xi$. Thus, (i) and (ii) are also satisfied in these cases.

For an LC network, $M(s)=T_{-1} s^{-1}+T_{1} s$ where $T_{-1}$ and $T_{1}$ are real-valued matrices with $T_{-1}, T_{1} \geq 0$. We note that $M(s)=-M(-s)^{T}$ and so $M^{-1}(s)=-\left(M^{-1}(-s)\right)^{T}$ and $M(j \omega)+M(j \omega)^{*}=0$ for all $0 \neq \omega \in \mathbb{R}$. It then follows from Section 2 that $M$ is $\mathrm{PR}$, whence $M^{-1}$ is $\mathrm{PR}$ by $\left[8\right.$, Theorem 5.8]. In particular, $M^{-1}$ has no poles in $\mathbb{C}_{+}$. Moreover, as $M^{-1}(s)=-\left(M^{-1}(-s)\right)^{T}$, we conclude that all of the poles of $M^{-1}$ must be on $j \mathbb{R} \cup \infty$.

Consider next an RC network. Then $M(s)=T_{0}+T_{1} s$ where $T_{0}$ and $T_{1}$ are real-valued matrices with $T_{0}, T_{1} \geq 0$. Accordingly, we define $\hat{M}(s):=M\left(s^{2}\right) / s$, and from the preceding paragraph it follows that $\hat{M}$ is $\mathrm{PR}$ and invertible, that $\hat{M}^{-1}$ is PR, and that all of the poles of $\hat{M}^{-1}$ are on $j \mathbb{R} \cup \infty$. Hence, from Section 2, the poles of $\hat{M}^{-1}$ are all simple and have positive semidefinite residue matrices. Furthermore, $\hat{M}^{-1}$ is real-rational and symmetric since $\hat{M}$ is, and it follows that all of the residue matrices of $\hat{M}^{-1}$ are symmetric, and that the poles and corresponding residue matrices of $\hat{M}^{-1}$ occur in complex conjugate pairs. It thus follows that $\hat{M}^{-1}$ has the partial fraction expansion $\hat{M}^{-1}(s)=\hat{W}_{\infty} s+\hat{W}_{0} / s+\sum_{i=1}^{n} \hat{W}_{i} /\left(s+j \omega_{i}\right)+\hat{W}_{i} /\left(s-j \omega_{i}\right)$ for some $\omega_{i} \in \mathbb{R}, \omega_{i}>0$, and some real-valued matrices $\hat{W}_{0}, \hat{W}_{\infty}, \hat{W}_{i} \geq 0(i=1, \ldots, n)$. Then, letting $\alpha_{i}:=$ $\omega_{i}^{2}$, we obtain $\hat{M}^{-1}(s)=s M^{-1}\left(s^{2}\right)=\hat{W}_{\infty} s+\hat{W}_{0} / s+$ $\sum_{i=1}^{n} 2 \hat{W}_{i} s /\left(s^{2}+\alpha_{i}\right)$, and so $M^{-1}(s)=\hat{W}_{\infty}+\hat{W}_{0} / s+$ $\sum_{i=1}^{n} 2 \hat{W}_{i} /\left(s+\alpha_{i}\right)$ for some $\alpha_{i}>0$ and real-valued matrices $\hat{W}_{0}, \hat{W}_{\infty}, \hat{W}_{i} \geq 0(i=1, \ldots, n)$. Hence, all of the poles 
of $M^{-1}$ are simple and have positive semidefinite residue matrices.

Finally, for an RL network, $M(s)=T_{-1} s^{-1}+T_{0}$ where $T_{-1}$ and $T_{0}$ are real-valued matrices with $T_{-1}, T_{0} \geq 0$. By a similar argument to before, this time considering $\bar{M}(s):=s M\left(s^{2}\right)$, we obtain $M^{-1}(s)=\bar{W}_{\infty} s+\bar{W}_{0}+$ $\sum_{i=1}^{n} 2 \bar{W}_{i} s /\left(s+\beta_{i}\right)$ for some $\beta_{i}>0$ and real-valued matrices $\bar{W}_{0}, \bar{W}_{\infty}, \bar{W}_{i} \geq 0(i=1,2, \ldots, n)$. We conclude that the poles of $M^{-1}$ in $\mathbb{C}_{-}$are at $-\beta_{1},-\beta_{2}, \ldots,-\beta_{n}$. Moreover, we find that $\lim _{s \rightarrow-\beta_{i}}\left(\left(s+\beta_{i}\right)^{k} M^{-1}(s)\right)=0$ whenever $k>1$, and $\lim _{s \rightarrow-\beta_{i}}\left(\left(s+\beta_{i}\right) M^{-1}(s)\right)=-2 \bar{W}_{i} \beta_{i}$, and hence the pole of $M^{-1}$ at $-\beta_{i}$ is simple and its residue matrix is negative semidefinite $(i=1,2, \ldots, n)$. This completes the proof of the present theorem.

Proof (Theorem 4). We recall from Section 3 that, up to a reordering of the variables, $\mathcal{B}=\left\{\mathbf{w} \in \mathcal{L}_{1}^{\text {loc }}\left(\mathbb{R}, \mathbb{R}^{2 m+2}\right)\right.$ $\left.W\left(\frac{d}{d t}\right) \mathbf{w}=0\right\}$ for some $W \in \mathbb{R}^{(2 m+1) \times(2 m+2)}[s]$ which takes the form of (9). With $K \in \mathbb{R}^{(m+1) \times m}$ and $L \in$ $\mathbb{R}^{m \times(m+2)}$ as in (10), we define $X \in \mathbb{R}^{(2 m+2) \times(2 m+1)}(s)$ as

$X:=\left[\begin{array}{cc}Y & 0 \\ -L Y & I\end{array}\right]\left[\begin{array}{cc}I & -K \\ 0 & I\end{array}\right]$, for $Y:=\left[\begin{array}{cc}0 & 0 \\ 1 & -x^{T} A^{-1} F^{-1} \\ 0 & A^{-1} F^{-1}\end{array}\right]$

From the proof of Theorem 2, $A^{-1} F^{-1}$ and hence also $X$ has no poles in $\mathbb{C}_{+}$, and any poles on $j \mathbb{R}$ are simple. Note that $R Y=I_{m+1}$ from (11), whence $W X=I_{2 m+1}$ from (10). In particular, $W(\xi)$ has rank $2 m+1$ for almost all $\xi \in \mathbb{C}$. Let $\lambda_{1}, \ldots, \lambda_{2 m+1} \in \mathbb{R}[s]$ denote the invariant polynomials of $W$ and let $U, V$ be unimodular and such that $U W V=\left[\begin{array}{ll}\Lambda & 0\end{array}\right]$ is in Smith form with $\Lambda=$ $\operatorname{diag}\left(\begin{array}{lll}\lambda_{1} & \cdots & \lambda_{2 m+1}\end{array}\right)$. Then $\Lambda^{-1}=\left[\begin{array}{ll}I_{2 m+1} & 0\end{array}\right] V^{-1} X U^{-1}$ and, since $U$ and $V$ are unimodular, we conclude that the roots of $\lambda_{k}$ are all in $\overline{\mathbb{C}}_{-}$and those on $j \mathbb{R}$ have multiplicity one $(k=1, \ldots, 2 m+1)$.

Now, let $\mathbf{w} \in \mathcal{B}$. Following [11, proof of Theorem $5.2 .14]$, we define

$$
R_{c}:=\left[\begin{array}{ll}
I_{2 m+1} & 0
\end{array}\right] V^{-1}, \text { and } R_{a}:=\left[\begin{array}{cc}
\Lambda & 0 \\
0 & 1
\end{array}\right] V^{-1} .
$$

Then $\mathbf{w}=\mathbf{w}_{1}+\mathbf{w}_{2}$ for $\mathbf{w}_{1} \in \mathcal{B}_{c}$ and $\mathbf{w}_{2} \in \mathcal{B}_{a}$, where

$$
\begin{aligned}
\mathcal{B}_{c} & :=\left\{\mathbf{w} \in \mathcal{L}_{1}^{\text {loc }}\left(\mathbb{R}, \mathbb{R}^{2 m+2}\right) \mid R_{c}\left(\frac{d}{d t}\right) \mathbf{w}=0\right\}, \\
\text { and } \mathcal{B}_{a} & :=\left\{\mathbf{w} \in \mathcal{L}_{1}^{\text {loc }}\left(\mathbb{R}, \mathbb{R}^{2 m+2}\right) \mid R_{a}\left(\frac{d}{d t}\right) \mathbf{w}=0\right\} .
\end{aligned}
$$

Since $V$ is unimodular, then the rank of $R_{c}(\xi)$ is the same for all $\xi \in \mathbb{C}$, and so $\mathcal{B}_{c}$ is controllable. Hence, there exists a $\hat{\mathbf{w}}_{1} \in \mathcal{B}_{c}$ and a $t_{1} \geq 0$ such that $\hat{\mathbf{w}}_{1}(t)=\mathbf{w}_{1}(t)$ for all $t \leq 0$, and $\hat{\mathbf{w}}_{1}(t)=0$ for all $t \geq t_{1}$ (see Section 2). Accordingly, to show the present theorem, it suffices to show that $\mathbf{w}_{2}(t)$ is bounded in $t \geq t_{1}$ whenever $\mathbf{w}_{2} \in \mathcal{B}_{a}$. Note initially that $R_{a}$ is square with $\operatorname{det}\left(R_{a}\right)=$ $\operatorname{det}(\Lambda) / \operatorname{det}(V) \not \equiv 0$, and so $\mathcal{B}_{a}=\mathcal{B}_{a} \cap \mathcal{C}^{\infty}\left(\mathbb{R}, \mathbb{R}^{2 m+2}\right)[11$, Section 3.2]. From (19), $\mathbf{w}_{2} \in \mathcal{B}_{a} \cap \mathcal{C}^{\infty}\left(\mathbb{R}, \mathbb{R}^{2 m+2}\right)$ if and only if $\mathbf{w}_{2}=V\left(\frac{d}{d t}\right) \hat{\mathbf{w}}$ for some $\hat{\mathbf{w}} \in \mathcal{C}^{\infty}\left(\mathbb{R}, \mathbb{R}^{2 m+2}\right)$ of the form $\hat{\mathbf{w}}=\operatorname{col}\left(\hat{w}_{1} \cdots \hat{w}_{2 m+2}\right)$, where $\hat{w}_{2 m+2}=0$, and $\lambda_{k}\left(\frac{d}{d t}\right) \hat{w}_{k}=0$ for $k=1, \ldots, 2 m+1$. Since the roots of $\lambda_{k}$ are all in $\overline{\mathbb{C}}_{-}$and those on $j \mathbb{R}$ have multiplicity one, then $\frac{d^{l} \hat{w}_{k}}{d t^{l}}(t)$ is bounded in $t \geq t_{1}(k=1, \ldots, 2 m+1$, and $l=1,2, \ldots)$. By denoting the $k$ th column of $V$ by $\mathbf{v}_{k}$, we obtain $\mathbf{w}_{2}=V\left(\frac{d}{d t}\right) \hat{\mathbf{w}}=\sum_{k=1}^{2 m+1} \mathbf{v}_{k}\left(\frac{d}{d t}\right) \hat{w}_{k}$, and so $\mathbf{w}_{2}(t)$ is also bounded in $t \geq t_{1}$.

\section{Sinusoidal trajectories and phasor analysis}

A classical analysis technique for RLC networks is the phasor approach. In this approach, network trajectories are considered in which the currents and voltages vary sinusoidally at a given frequency $\omega$, and these are used to define the 'ac-steady-state impedance' [13, Section 5.4]. In [13, p. 167] it is shown that, providing the 'loop impedance matrix' is nonsingular on the entire imaginary axis, then 1) the 'ac-steady-state impedance' is closely related to the impedance of the network, and 2) a similar relationship 'applies to all the other network functions'. However, it should be noted that there can be points on the imaginary axis where the 'loop impedance matrix' is singular. This is the case at $s= \pm j$ for central network in Fig. 3. As will be shown in this section, condition 1 still holds in this case, but condition 2 is no longer true.

For $\omega \in \mathbb{R}$, we define a sinusoidal trajectory of an RLC network as a full trajectory $\mathbf{b}$ of the network for which

$$
\mathbf{b}(t)=\Re\left(\operatorname{col}\left(\begin{array}{llllllll}
\tilde{i} & \tilde{v} & \tilde{i}_{1} & \cdots & \tilde{i}_{m} & \tilde{v}_{1} & \cdots & \tilde{v}_{m}
\end{array}\right) e^{j \omega t}\right),
$$

for all $t \in \mathbb{R}$, and with $\tilde{i}, \tilde{v}, \tilde{i}_{k}, \tilde{v}_{k} \in \mathbb{C}(k=1, \ldots, m)$. The main result in this section is the following theorem:

Theorem 5. Let $N$ be an RLC network with impedance $Z$, and let $\omega \in \mathbb{R}$. Then $N$ possesses a sinusoidal trajectory at frequency $\omega$ as in (20) for which at least one of $\tilde{i}$ or $\tilde{v}$ is non-zero. Moreover, for any such trajectory, either $\tilde{i}$ is identically zero and $Z$ has a pole at $j \omega$, or $Z(j \omega)=\tilde{v} / \tilde{i}$.

To illustrate Theorem 5, we consider again the three networks in Fig. 3. It may be verified that $\mathbf{b}_{1}, \mathbf{b}_{2}$, and $\mathbf{b}_{3}$ are sinusoidal trajectories at frequency 1 for the left-hand, central, and right-hand networks in Fig. 3, respectively, where $\mathbf{b}_{1}(t)=\Re\left(\tilde{i} \tilde{\mathbf{c}}_{1} e^{j t}\right), \mathbf{b}_{2}(t)=\Re\left(\left(\tilde{i} \tilde{\mathbf{c}}_{2}+\tilde{i}_{1} \tilde{\mathbf{c}}_{3}\right) e^{j t}\right)$, and $\mathbf{b}_{3}(t)=\Re\left(\left(\tilde{i} \tilde{\mathbf{c}}_{4}+\tilde{i}_{1} \tilde{\mathbf{c}}_{\mathbf{5}}\right) e^{j t}\right)$ for all $t \in \mathbb{R}$ and for any $\tilde{i}, \tilde{i}_{1} \in$ $\mathbb{C}$, where $\tilde{\mathbf{c}}_{1}, \tilde{\mathbf{c}}_{2}, \tilde{\mathbf{c}}_{3}, \tilde{\mathbf{c}}_{4}$, and $\tilde{\mathbf{c}}_{5}$ are given by

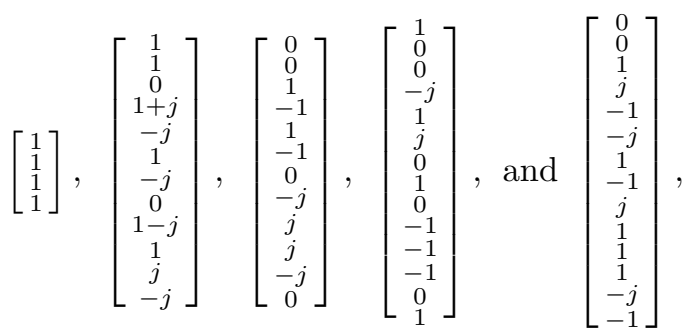

respectively. For the two leftmost networks, whenever $\tilde{i} \neq 0$, we have $\tilde{v} / \tilde{i}=1=Z(j)$. However, for the righthand network (which contains a resistor with a negative 
resistance), whenever $\tilde{i} \neq 0$, we have $\tilde{v} / \tilde{i}=0 \neq Z(j)$, and Theorem 5 fails to hold.

The central network in Fig. 3 is not stabilisable, and so a given driving-point current $i\left(i(t)=\Re\left(\tilde{i} e^{j t}\right)\right.$ for all $t \in$ $\mathbb{R})$ does not determine a unique sinusoidal trajectory. In particular, the ratio of internal currents and voltages need not equal the value of the corresponding transfer function at $j$. For example, the transfer function $H \in \mathbb{R}(s)$ from $i$ to $v_{1}$ satisfies $H(j) \neq 0$, yet for the trajectory $\mathbf{b}_{2}$ with $\mathbf{b}_{2}(t)=\Re\left(\tilde{\mathbf{c}}_{2} e^{j t}\right)$ for all $t \in \mathbb{R}$ we have $\tilde{v}_{1} / \tilde{i}=0$.

Proof (Theorem 5). The driving-point behavior of $N$ takes the form indicated in Theorem 2, where $f(j \omega) \neq 0$, and either $p(j \omega) \neq 0$ or $q(j \omega) \neq 0$. Hence, $Z=p / q$ has a pole at $j \omega$ if and only if $q(j \omega)=0$, and whenever $i(t)=\Re\left(\tilde{i} e^{j \omega t}\right)$ and $v(t)=\Re\left(\tilde{v} e^{j \omega t}\right)$ for all $t \in \mathbb{R}$, then $p(j \omega) \tilde{i}=q(j \omega) \tilde{v}$. Thus, if $\tilde{i}=0$ and $\tilde{v} \neq 0$, then $q(j \omega)=0$ (so $Z$ has a pole at $j \omega$ ). If, on the other hand, $\tilde{i} \neq 0$, then $q(j \omega) \neq 0$, and $\tilde{v} / \tilde{i}=p(j \omega) / q(j \omega)=Z(j \omega)$.

It remains to show that $N$ possesses a sinusoidal trajectory at frequency $\omega$ as in (20) for which at least one of $\tilde{i}$ or $\tilde{v}$ is non-zero. From the discussion in Section 3, it suffices to show that there is a non-zero vector in the kernel of $R(j \omega)$ for $R$ as in (11), and for which at least one of the first two entries is non-zero. Accordingly, we let

$$
z:=\operatorname{col}\left(\begin{array}{lll}
h & x^{T} g & -g
\end{array}\right), \text { where } A^{-1} x=g h^{-1},
$$

for $g h^{-1}$ a right coprime factorisation over $\mathcal{R} \mathcal{H}_{\infty}$ of $A^{-1} x$. This implies that $g \in \mathbb{R}_{p}^{m}(s)$ and $h \in \mathbb{R}_{p}(s)$, and that $z$ is analytic and non-zero in $\bar{C}_{+} \cup \infty$. Furthermore, $R z=0$ from (11), so $R(j \omega) z(j \omega)=0$ for any given $\omega \in \mathbb{R}$, and it suffices to show that either $h(j \omega)$ or $x^{T} g(j \omega)$ is non-zero.

We note from (21) that $x^{T} g=x^{T} A^{-1} x h$. By a similar argument to the proof of Theorem 2 , then $h(j \omega)=0$ and $x^{T} g(j \omega)=0$ together imply that $x^{T} A^{-1} x$ does not have a pole at $j \omega$, whence $A^{-1} x$ does not have a pole at $j \omega$, and so $A^{-1} x h(j \omega)=g(j \omega)=0$. This implies that $z(j \omega)=0$, which is not possible, and we conclude that either $h(j \omega)$ or $x^{T} g(j \omega)$ is non-zero.

\section{Elimination of internal variables}

A simple extension of the proof of Theorem 5 will now be used to show Theorem 1.

Proof (Theorem 1). From the final two block rows in (7), it follows that col $\left(\begin{array}{ll}\mathbf{v}_{1} & \mathbf{i}_{2}\end{array}\right)$ is locally integrable whenever col $\left(\begin{array}{llll}i & v & \mathbf{i}_{1} & \mathbf{v}_{2}\end{array}\right)$ is. Thus, from [10, Theorem 2.5 and Lemma 2.7] and from (11) and (13), it suffices to show that whenever $\operatorname{col}\left(\begin{array}{llll}i & v & \mathbf{i}_{1} & \mathbf{v}_{2}\end{array}\right) \in \mathcal{L}_{1}^{\text {loc }}\left(\mathbb{R}, \mathbb{R}^{m+2}\right)$ satisfies $R_{1 a}\left(\frac{d}{d t}\right) i+R_{1 b}\left(\frac{d}{d t}\right) v+R_{2}\left(\frac{d}{d t}\right) \operatorname{col}\left(\mathbf{i}_{1} \quad \mathbf{v}_{2}\right)=0$, with $R_{1 a}:=$ $\operatorname{col}\left(\begin{array}{lll}0 & F x\end{array}\right)$ and $R_{1 b}:=\operatorname{col}\left(\begin{array}{ll}1 & 0\end{array}\right)$, then either (i) there exists a proper transfer function from $i$ to $\operatorname{col}\left(\begin{array}{lll}v & \mathbf{i}_{1} & \mathbf{v}_{2}\end{array}\right)$, or (ii) there exists a proper transfer function from $v$ to $\operatorname{col}\left(\begin{array}{lll}i & \mathbf{i}_{1} & \mathbf{v}_{2}\end{array}\right)$. Note that $\operatorname{det}\left(\left[\begin{array}{ll}R_{1 b} & R_{2}\end{array}\right]\right)=\operatorname{det}(F A)$,

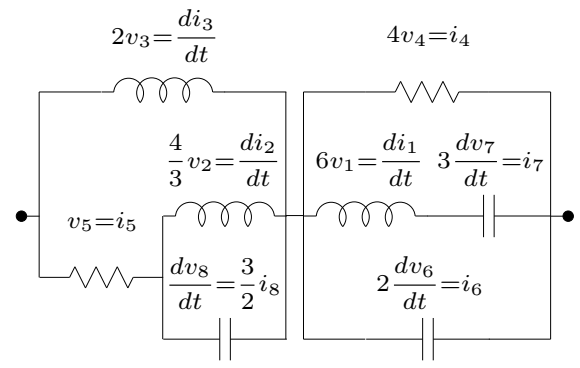

Figure 4: Bott-Duffin network for realising $\left(s^{2}+s+1\right) /\left(s^{2}+s+4\right)$

hence $\left[\begin{array}{ll}R_{1 b} & R_{2}\end{array}\right]$ is invertible since $F$ and $A$ are. Furthermore, $\operatorname{det}\left(\left[\begin{array}{ll}R_{1 a} & R_{2}\end{array}\right]\right)=-\operatorname{det}(F A) x^{T} A^{-1} x$, hence $\left[\begin{array}{ll}R_{1 a} & R_{2}\end{array}\right]$ is invertible whenever $x^{T} A^{-1} x \not \equiv 0$. Thus, it remains to show that either (i) $\left[\begin{array}{ll}R_{1 b} & R_{2}\end{array}\right]^{-1} R_{1 a} \in \mathbb{R}_{p}^{m+1}(s)$, or (ii) $x^{T} A^{-1} x \not \equiv 0$ with $\left[\begin{array}{ll}R_{1 a} & R_{2}\end{array}\right]^{-1} R_{1 b} \in \mathbb{R}_{p}^{m+1}(s)$.

We recall from the proof of Theorem 5 that $z$ in (21) is analytic and non-zero in $\bar{C}_{+} \cup \infty$ and satisfies $R z=$ 0 , whence $R_{1 a} h+R_{1 b} x^{T} g-R_{2} g=0$. Furthermore, a similar argument to the proof of that theorem shows that either $h(\infty) \neq 0$ or $x^{T} g(\infty) \neq 0$. If $h(\infty) \neq 0$, then $\left[\begin{array}{ll}R_{1 b} & R_{2}\end{array}{ }^{-1} R_{1 a}=-\operatorname{col}\left(x^{T} g-g\right) h^{-1} \in \mathbb{R}_{p}^{m+1}(s)\right.$. If, instead, $x^{T} g(\infty) \neq 0$, then $x^{T} A^{-1} x=x^{T} g h^{-1} \not \equiv 0$, and $\left[\begin{array}{ll}R_{1 a} & R_{2}\end{array}\right]^{-1} R_{1 b}=-\operatorname{col}(h-g)\left(x^{T} g\right)^{-1} \in \mathbb{R}_{p}^{m+1}(s)$.

\section{The Bott-Duffin networks}

The Bott-Duffin procedure [3] generates an RLC network to realise any given PR function. Ever since its publication, it has been a significant puzzle that the number of energy storage elements used by the procedure is considerably greater than the McMillan degree of the transfer function being realised. Despite this, it was shown in [7] that the Bott-Duffin networks actually contain the least possible number of energy storage elements for the realisation of a special class of PR functions (the biquadratic minimum functions) among all series-parallel networks.

In this section, we investigate the controllability of the driving-point and full behavior of the Bott-Duffin realisation for the biquadratic minimum function $Z(s):=$ $\left(s^{2}+s+1\right) /\left(s^{2}+s+4\right)$, which is shown in Fig. 4. Following Section 3, we find that the driving-point behavior of this network is the set of locally integrable functions in the kernel of $\left(\frac{d}{d t}+1\right)\left[-\left(\frac{d^{2}}{d t^{2}}+\frac{d}{d t}+1\right) \quad \frac{d^{2}}{d t^{2}}+\frac{d}{d t}+4\right]$. Moreover, the full behavior is the set of locally integrable functions in the kernel of a differential operator $W\left(\frac{d}{d t}\right)$ with $W \in$ $\mathbb{R}^{17 \times 18}[s]$ which has invariant polynomials $\lambda_{1}, \ldots, \lambda_{17}$ with $\lambda_{1}(s)=\ldots=\lambda_{16}(s)=1$, and $\lambda_{17}(s)=(s+1)\left(s^{2}+s+4\right)$. In other words, the driving-point behavior has a single uncontrollable mode, and there are further uncontrollable (but stabilisable) modes in the full behavior which are not observed at the driving-point terminals. 


\section{Stabilisability of passive behaviors}

In this section, we provide an alternative approach to, and generalisation of, Theorem 2. The essence of the approach is an energy (passivity) argument which does not require a detailed kernel representation of the network's behavior. The argument applies to the driving-point behavior of one-port RLC networks as well as the terminal behavior of general networks comprising the usual passive circuit elements (resistor, capacitor, inductor, transformer, gyrator) as considered in [17].

We consider a behavior $\mathcal{B}$ comprised of trajectories $\mathbf{d} \in$ $\mathcal{L}_{1}^{\text {loc }}\left(\mathbb{R}, \mathbb{R}^{2 N}\right)$ of the form:

$$
\begin{aligned}
\mathbf{d} & :=\operatorname{col}\left(\begin{array}{ll}
\mathbf{i} & \mathbf{v}
\end{array}\right), \\
\text { with } \mathbf{i}: & :=\operatorname{col}\left(\begin{array}{llll}
i_{1} & i_{2} & \cdots & i_{N}
\end{array}\right), \\
\text { and } \quad \mathbf{v} & :=\operatorname{col}\left(\begin{array}{llll}
v_{1} & v_{2} & \cdots & v_{N}
\end{array}\right) .
\end{aligned}
$$

Firstly, we specify that $\mathcal{B}$ has a hybrid representation (see [17, p. 24]). This implies that $\mathbf{i}$ and $\mathbf{v}$ may be partitioned compatibly as $\mathbf{i}:=\operatorname{col}\left(\begin{array}{ll}\mathbf{i}_{1} & \mathbf{i}_{2}\end{array}\right)$ and $\mathbf{v}:=\operatorname{col}\left(\begin{array}{ll}\mathbf{v}_{1} & \mathbf{v}_{2}\end{array}\right)$ such that $\mathcal{B}$ is the set of locally integrable solutions to

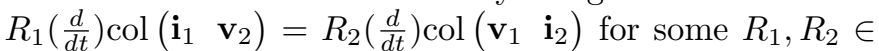
$\mathbb{R}^{N \times N}[s]$ with $R_{1}(\xi)$ invertible for almost all $\xi \in \mathbb{C}$.

Secondly, we call $\mathcal{B}$ passive if, for any trajectory in $\mathcal{B}$ and any $t_{0} \in \mathbb{R}$, there exists a $K \in \mathbb{R}$ (depending on the trajectory and $\left.t_{0}\right)$ such that $\int_{t_{0}}^{t_{1}} \sum_{k=1}^{N} i_{k}(t) v_{k}(t) d t>-K$ for all $t_{1} \geq t_{0}$. This has the interpretation that, for any starting time $t_{0}$, there is a limit to the net amount of energy that can be extracted from the network from $t_{0}$ onwards. It is pointed out in [17] that the terminal behavior of any resistor, inductor, capacitor, transformer, or gyrator has a hybrid representation and is passive, as does the terminal behavior of any interconnection of these elements. Similarly, the driving-point behavior of any network comprising these elements also has a hybrid representation and is passive [1, Section 2.3 and Lemma 4.4.1].

The main result in this section is the following: ${ }^{5}$

Theorem 6. Any passive behavior with a hybrid representation is stabilisable.

Proof. Since the behavior $\mathcal{B}$ admits a hybrid representation then it is the set of locally integrable functions in the kernel of a differential operator $R\left(\frac{d}{d t}\right)$ where $R \in \mathbb{R}^{N \times 2 N}[s]$ and $R(\xi)$ has rank $N$ for almost all $\xi \in \mathbb{C}$. Let us assume that $\mathcal{B}$ is not stabilisable. Then, from Section 2 , there exists a $\xi=\sigma+j \omega$ with $\sigma, \omega \in \mathbb{R}$ and $\sigma \geq 0$ such that the rank of $R(\xi)$ is less than $N$. We will show that this implies that $\mathcal{B}$ is not passive.

For $\omega \neq 0$ (resp. $\omega=0$ ), we consider the $\mathbb{C}$-vector space (resp. $\mathbb{R}$-vector space) corresponding to the solutions $\mathbf{w}$ to $R(\xi) \mathbf{w}=0$, which is a linear subspace of $\mathbb{C}^{2 N}\left(\right.$ resp. $\left.\mathbb{R}^{2 N}\right)$ of dimension greater than $N$. To begin with, we will show that any such space contains a vector $\tilde{\mathbf{d}}$ such that

$$
\Pi:=\tilde{\mathbf{d}}^{*}\left[\begin{array}{cc}
0 & I_{N} \\
I_{N} & 0
\end{array}\right] \tilde{\mathbf{d}}<0 .
$$

Then, we let $\mathbf{d}$ in $(22)$ satisfy $\mathbf{d}(t):=\Re\left(\tilde{\mathbf{d}} e^{(\sigma+j \omega) t}\right)$ for all $t \in \mathbb{R}$. Since $R(\xi) \tilde{\mathbf{d}}=0$, then $\mathbf{d}$ is a trajectory in $\mathcal{B}$. For this trajectory, we will show that there is a $t_{0} \in \mathbb{R}$ such that, for any $K \in \mathbb{R}$, there exists a $t_{1} \geq t_{0}$ for which $\int_{t_{0}}^{t_{1}} \sum_{k=1}^{N} i_{k}(t) v_{k}(t) d t<-K$, and hence $\mathcal{B}$ is not passive.

Firstly, for both the cases $\mathbb{F}=\mathbb{C}$ and $\mathbb{F}=\mathbb{R}$, we consider a linear subspace of $\mathbb{F}^{2 N}$ with dimension $N+r$ for some $r \geq 1$, and we let $Z \in \mathbb{F}^{2 N \times(N+r)}$ be a matrix whose columns are a basis for this subspace. Further, we partition $Z$ as $Z=$ : $\operatorname{col}\left(Z_{1} \quad Z_{2}\right)$ with $Z_{1}, Z_{2} \in \mathbb{F}^{N \times(N+r)}$, and we define $X_{1}$ and $X_{2}$ by the invertible transformation:

$$
\left[\begin{array}{l}
X_{1} \\
X_{2}
\end{array}\right]:=\frac{1}{\sqrt{2}}\left[\begin{array}{cc}
I_{N} & I_{N} \\
I_{N} & -I_{N}
\end{array}\right]\left[\begin{array}{l}
Z_{1} \\
Z_{2}
\end{array}\right]
$$

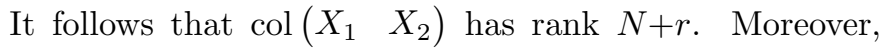
$X_{1} \in \mathbb{F}^{N \times(N+r)}$, so the rank of $X_{1}$ is at most $N$, and hence there exists a $\mathbf{y} \in \mathbb{F}^{N+r}$ such that $X_{1} \mathbf{y}=0$ but $\mathbf{w}:=X_{2} \mathbf{y} \neq 0$. Then defining

$$
\tilde{\mathbf{d}}:=\left[\begin{array}{c}
Z_{1} \\
Z_{2}
\end{array}\right] \mathbf{y}=\frac{1}{\sqrt{2}}\left[\begin{array}{cc}
I_{N} & I_{N} \\
I_{N} & -I_{N}
\end{array}\right]\left[\begin{array}{l}
X_{1} \\
X_{2}
\end{array}\right] \mathbf{y}=\frac{1}{\sqrt{2}}\left[\begin{array}{c}
\mathbf{w} \\
-\mathbf{w}
\end{array}\right],
$$

which is in the column space of $Z$, we have

$$
\tilde{\mathbf{d}}^{*}\left[\begin{array}{cc}
0 & I_{N} \\
I_{N} & 0
\end{array}\right] \tilde{\mathbf{d}}=\frac{1}{2}\left[\begin{array}{ll}
\mathbf{w}^{*} & -\mathbf{w}^{*}
\end{array}\right]\left[\begin{array}{c}
-\mathbf{w} \\
\mathbf{w}
\end{array}\right]=-\mathbf{w}^{*} \mathbf{w}<0 .
$$

We conclude that there is a vector

$$
\tilde{\mathbf{d}}=: \operatorname{col}\left(\begin{array}{llllllll}
\tilde{i}_{1} & \tilde{i}_{2} & \cdots & \tilde{i}_{N} & \tilde{v}_{1} & \tilde{v}_{2} & \cdots & \tilde{v}_{N}
\end{array}\right) \in \mathbb{F}^{2 N}
$$

satisfying $R(\xi) \tilde{\mathbf{d}}=0$ and the inequality (23), and we note that $\Pi=2 \sum_{k=1}^{N} \Re\left(\tilde{i}_{k}^{*} \tilde{v}_{k}\right)$. We now let $\mathbf{d}$ in (22) satisfy $\mathbf{d}(t):=\Re\left(\tilde{\mathbf{d}} e^{(\sigma+j \omega) t}\right)$ for all $t \in \mathbb{R}$, so $\mathbf{d} \in \mathcal{B}$, and we consider $\int_{t_{0}}^{t_{1}} \sum_{k=1}^{N} i_{k}(t) v_{k}(t) d t$.

Suppose initially that $\omega \neq 0$ and $\tilde{\mathbf{d}} \in \mathbb{C}^{2 N}$. Without loss of generality, we let $\omega>0$. Let $t_{1}=t_{0}+(n \pi / \omega)$ with $n \in \mathbb{Z}$. For $\sigma>0, \int_{t_{0}}^{t_{0}+(n \pi / \omega)} \sum_{k=1}^{N} i_{k}(t) v_{k}(t) d t$ is equal to

$$
\begin{aligned}
& \frac{1}{2} \Re \int_{t_{0}}^{t_{0}+(n \pi / \omega)}\left(\sum_{k=1}^{N} \tilde{i}_{k} \tilde{v}_{k} e^{2(\sigma+j \omega) t}+\sum_{k=1}^{N} \tilde{i}_{k}^{*} \tilde{v}_{k} e^{2 \sigma t}\right) d t \\
& =\frac{e^{2 \sigma t_{0}}}{4}\left(e^{2 \sigma n \pi / \omega}-1\right) \Re\left(\frac{e^{2 j \omega t_{0}}}{\sigma+j \omega} \sum_{k=1}^{N} \tilde{i}_{k} \tilde{v}_{k}+\frac{1}{\sigma} \sum_{k=1}^{N} \tilde{i}_{k}^{*} \tilde{v}_{k}\right) .
\end{aligned}
$$

For any $\tilde{\mathbf{d}} \in \mathbb{C}^{2 N}$, there exists a $t_{0} \in \mathbb{R}$ with $0 \leq t_{0} \leq \pi / \omega$ such that $\Re\left(e^{2 j \omega t_{0}} \sum_{k=1}^{N} \tilde{i}_{k} \tilde{v}_{k} /(\sigma+j \omega)\right)=0$, in which case $\int_{t_{0}}^{t_{0}+(n \pi / \omega)} \sum_{k=1}^{N} i_{k}(t) v_{k}(t) d t=e^{2 \sigma t_{0}}\left(e^{2 \sigma n \pi / \omega}-1\right) \Pi /(8 \sigma)$, where $\Pi$ is as defined in (23). For $\sigma=0$, we consider $2 \int_{t_{0}}^{t_{0}+(n \pi / \omega)} \sum_{k=1}^{N} i_{k}(t) v_{k}(t) d t$, and we find this to equal $\Re \int_{t_{0}}^{t_{0}+(n \pi / \omega)}\left(\sum_{k=1}^{N} \tilde{i}_{k} \tilde{v}_{k} e^{2 j \omega t}+\sum_{k=1}^{N} \tilde{i}_{k}^{*} \tilde{v}_{k}\right) d t=n \pi \Pi /(2 \omega)$. In both cases, by choosing $n$ sufficiently large, we can ensure $\int_{t_{0}}^{t_{0}+(n \pi / \omega)} \sum_{k=1}^{N} i_{k}(t) v_{k}(t) d t<-K$ for any $K \in \mathbb{R}$.

Suppose, instead, that $\omega=0$ and $\tilde{\mathbf{d}} \in \mathbb{R}^{2 N}$. In this case, $\int_{t_{0}}^{t_{1}} \sum_{k=1}^{N} i_{k}(t) v_{k}(t) d t=\int_{t_{0}}^{t_{1}} \sum_{k=1}^{N} \tilde{i}_{k} \tilde{v}_{k} e^{2 \sigma t} d t$, which equals $\left(e^{2 \sigma t_{1}}-e^{2 \sigma t_{0}}\right) \Pi /(4 \sigma)$ when $\sigma>0$, and $\left(t_{1}-t_{0}\right) \Pi / 2$ when $\sigma=0$. Again, given any $K \in \mathbb{R}$, then there exists a $t_{1} \geq t_{0}$ such that $\int_{t_{0}}^{t_{1}} \sum_{k=1}^{N} i_{k}(t) v_{k}(t) d t<-K$. 


\section{Conclusions}

This paper has been concerned with fundamental aspects of RLC networks, in particular, the relationship between the driving-point and the full behavior, the role of controllability, and the rigorous formulation of phasor analysis. Our approach provides ample illustration of the vast legacy of Jan Willems. Three of his major ideas have played a pivotal role in our approach: (1) terminals and ports, (2) passivity and dissipativity, and (3) behaviors.

\section{Notes}

1. By a graph we mean an ordered pair $(V, E)$ where $V$ is a set $\left\{x_{1}, \ldots, x_{n}\right\}$ whose elements are called vertices and $E$ is a set $\left\{y_{1}, \ldots, y_{q}\right\}$ of unordered pairs of vertices called edges, i.e. $y_{k}=\left(x_{k_{1}}, x_{k_{2}}\right), k=1, \ldots, q$ [2]. Our definition of connected follows [2]. In contrast to [2], we allow several edges to join the same two vertices. A graph is called oriented when each edge has one of its vertices arbitrarily assigned as a head vertex and the other as a tail vertex.

2. A circuit in a graph is a sequence of distinct edges from a vertex to itself with no other vertices repeated. A tree (called spanning tree in [2]) in a connected graph is a connected subgraph containing all of the vertices and no circuit. A chord-set in a connected graph is a subset of its edges such that the complementary set of edges form a tree. If a connected graph $G$ comprises $n$ vertices and $q$ edges, then any tree (resp. chord-set) in $G$ contains exactly $n-1$ (resp. $q+1-n$ ) edges [2]. For a connected graph, a cut is a set of edges whose removal partitions the vertices into two disjoint sets. A cut-set is a cut for which no proper subset of the edges in the cut is also a cut.

3. Since the dimension of the cut-set (resp. circuit) space is $n-1$ (resp. $m+2-n$ ), then these spaces are orthogonal complements in $\mathbb{R}^{m+1}$. It follows that $\mathbf{b}$ in $(2)$ is a full trajectory of the network if and only if $i_{k}$ and $v_{k}$ satisfy the differential equation $p_{k}\left(\frac{d}{d t}\right) i_{k}=q_{k}\left(\frac{d}{d t}\right) v_{k}$ corresponding to the element $N_{k}(k=1, \ldots, m)$, and $\mathbf{i}(t)$ (resp. $\mathbf{v}(t))$ is in the circuit (resp. cut-set) space of $G^{\prime}$ for all $t \in \mathbb{R}$. In particular, given the orthogonality of these spaces, we have $\mathbf{i}^{T} \mathbf{v} \equiv 0$ whenever $\mathbf{i}$ satisfies Kirchhoff's current law and v satisfies Kirchhoff's voltage law. This property is known as Tellegen's theorem.

4. From the explicit description in Section 3 for the driving-point behavior of an RLC network, it is evident that the impedance of any (connected) RLC network exists and, in the terminology of that section, is equal to $Z=r^{-1} y^{T} F x=x^{T} A^{-1} x$, which is $\mathrm{PR}$ since $A^{-1}$ is $\mathrm{PR}$ [8, Theorem 5.5]. Moreover, whenever the driving-point terminals of the network are distinct, then $C_{1} \neq 0$, which implies $x \neq 0$, and it follows that $Z \not \equiv 0$ as $A^{-1}$ is PR.
5. We note that the assumption in Theorem 6 of existence of a hybrid representation cannot be simply omitted. This is demonstrated by the full behavior of the central network in Fig. 3. From note 3, it follows that $\int_{t_{0}}^{t_{1}} \mathbf{i}(t)^{T} \mathbf{v}(t) d t=0$ for all $t_{0}, t_{1} \in \mathbb{R}$, and hence $\int_{t_{0}}^{t_{1}} \mathbf{i}(t)^{T} \mathbf{v}(t) d t>-K$ for all $t_{0}, t_{1} \in \mathbb{R}$ and for any choice of $K>0$. However, as shown in Section 4 , the full behavior of this network is not stabilisable. Indeed, if a behavior $\mathcal{B}$ comprised of trajectories $\mathbf{d} \in \mathcal{L}_{1}^{\text {loc }}\left(\mathbb{R}, \mathbb{R}^{2 N}\right)$ of the form of (22) does not have a hybrid representation, then for any $\mathbf{d} \in \mathcal{B}$ there can exist a $K \in \mathbb{R}$ such that $\int_{t_{0}}^{t_{1}} \sum_{k=1}^{N} i_{k}(t) v_{k}(t) d t>$ $-K$ for all $t_{0}, t_{1} \in \mathbb{R}$, and yet $\mathcal{B}$ need not even be marginally stabilisable. One such example corresponds to the network obtained by substituting $-\frac{d}{d t}$ for $\frac{d}{d t}$ in the central network in Fig. 3 .

[1] B. D. O. Anderson and S. Vongpanitlerd. Network Analysis and Synthesis. Upper Saddle River, NJ: Prentice-Hall, 1973.

[2] Béla Bollobás. Modern graph theory. New York: Springer, 1998.

[3] R. Bott and R. J. Duffin. Impedance synthesis without use of transformers. J. Appl. Phys., 20:816, 1949.

[4] O. Brune. Synthesis of a finite two-terminal network whose driving-point impedance is a prescribed function of frequency. J. Math. Phys., 10:191-236, 1931.

[5] A. Fialkow and I. Gerst. Impedance synthesis without mutual coupling. Quart. Appl. Math., 12:420-422, 1955.

[6] T. H. Hughes. Why RLC realizations of certain impedances require a majority of uncontrollable or unobservable modes. Submitted to IEEE Trans. on Automatic Control, 2015.

[7] T. H. Hughes and M. C. Smith. On the minimality and uniqueness of the Bott-Duffin realization procedure. IEEE Trans. on Automatic Control, 59(7):1858-1873, July 2014.

[8] Robert W. Newcomb. Linear Multiport Synthesis. McGraw Hill, 1966.

[9] R. H. Pantell. A new method of driving point impedance synthesis. Proc. IRE (Correspondence), 42:861, 1954.

[10] J. W. Polderman. Proper elimination of latent variables. Systems and Control Letters, 32:262-269, 1997.

[11] J. W. Polderman and J. C. Willems. Introduction to Mathematical Systems Theory: A Behavioral Approach. New York: Springer-Verlag, 1998.

[12] F. M. Reza. Synthesis without ideal transformers. J. Appl. Phys., 25:807-808, March 1954.

[13] S. Seshu and N. Balabanian. Linear Network Analysis. John Wiley \& Sons, 1959.

[14] J. E. Storer. Relationship between the Bott-Duffin and Pantell impedance synthesis. Proc. IRE, 42:1451, September 1954.

[15] J. C. Willems. The behavioral approach to open and interconnected systems. IEEE Control Systems Magazine, 27:46-99, 2007.

[16] J. C. Willems. Dissipative dynamical systems. European Journal on Control, 13:134-151, 2007.

[17] J. C. Willems. Terminals and ports. IEEE Circuit and Systems Magazine, 10(4):8-26, Dec. 2010.

[18] D. C. Youla and P. Tissi. N-port synthesis via reactance extraction, Part I. IEEE International Convention Record, 14(7):183205, 1966. 